\title{
Reactionary Responses to the Bad Lot Objection
}

\author{
Finnur Dellsén \\ Preprint; please cite published version in Studies in History and Philosophy of Science
}

\begin{abstract}
As it is standardly conceived, Inference to the Best Explanation (IBE) is a form of ampliative inference in which one infers a hypothesis because it provides a better potential explanation of one's evidence than any other available, competing explanatory hypothesis. Bas van Fraassen famously objected to IBE thus formulated that we may have no reason to think that any of the available, competing explanatory hypotheses are true. While revisionary responses to the Bad Lot Objection concede that IBE needs to be reformulated in light of this problem, reactionary responses argue that the Bad Lot Objection is fallacious, incoherent, or misguided. This paper shows that the most influential reactionary responses to the Bad Lot Objection do nothing to undermine the original objection. This strongly suggests that proponents of IBE should focus their efforts on revisionary responses, i.e. on finding a more sophisticated characterization of IBE for which the Bad Lot Objection loses its bite.
\end{abstract}

Keywords: Inference to the Best Explanation; the Bad Lot Objection; underconsideration; ranking of theories; inductive cogency.

\section{Introduction}

As it is standardly conceived, Inference to the Best Explanation (IBE) is a form of ampliative inference in which one infers a hypothesis because it provides a better potential explanation of one's evidence than any other available, competing explanatory hypothesis. Bas van Fraassen (1989: 142-143) famously objected to IBE thus formulated that we may have no reason to think that any of the available, competing explanatory hypotheses are true. The hypotheses one has so far generated (i.e. made available) may all be false, in which case the true explanation would be provided by a hypothesis outside of the set of available hypotheses. In that case, IBE would lead us to a false conclusion, no matter how 
good we are at finding the best explanatory hypothesis among the available competitors. This Bad Lot Objection is often considered to be one of the main threats to IBE as an cogent form of ampliative inference; as such, it has shaped much of the debate about IBE in the last few decades. ${ }^{1}$

Responses to the Bad Lot Objection fall into two broad categories. On the one hand, van Fraassen's objection has prompted some philosophers to find a more sophisticated characterization of IBE for which the objection does not arise. Such a revisionary response can be achieved in various ways - by adding some further conditions on the applicability of IBE (e.g. by adding the restriction that the best explanation must also meet some minimal requirements of explanatory goodness); by weakening the form of the conclusion (e.g. by replacing truth simpliciter with approximate truth, probable truth, or probable approximate truth); or indeed by re-conceiving of IBE as playing merely an auxiliary role in some other epistemological framework (e.g. probabilistic or 'Bayesian' epistemology). ${ }^{2}$ On the other hand, some philosophers have responded to the Bad Lot Objection by attacking van Fraassen's argument directly - arguing that van Fraassen has not identified a reason to revise or reformulate our original definition of IBE. These reactionary responses are the topic of this paper.

I will discuss what I consider to be the three most prominent responses of this kind - two responses due to the most influential proponent of IBE, Peter Lipton (1993; 2004: 151-163), and a more recent response from a leading member of a new generation of IBE-

1 The objection is also known as the Argument from Underconsideration (see, e.g., Lipton 1993, 2004; Wray 2008; Khalifa 2010). A related issue is the Problem of Unconceived Alternatives (PUA; see Sklar 1981, Stanford 2006, Wray 2011) - a problem for scientific realism motivated by a historical induction similar to the famous Pessimistic Meta-Induction (Poincaré 1952, Laudan 1981, Cassirer 1991). I will not be directly discussing PUA here, since (i) my focus here is on the purely epistemological issue of how to conceive of IBE rather than its application in the scientific realism debate, and (ii) PUA has been discussed at length elsewhere (see, e.g., Chakravartty 2008, Magnus 2010, Devitt 2011, Egg 2016, Dellsén forthcoming).

2 Indeed, van Fraassen (1989: 145-170) famously considers such a role for IBE at length within a Bayesian framework, although he ends up rejecting that IBE can play even this more modest role. Other authors have been more optimistic, either because they reject van Fraassen's argument (e.g., Douven 1999, 2013) or because they reject van Fraassen's conception of how to locate IBE in a Bayesian framework (e.g., Okasha 2000, Weisberg 2009, Henderson 2014). 
advocates, Jonah Schupbach (2014). ${ }^{3,4}$ Lipton's first argument is that the Bad Lot Objection can always be avoided since any comparative evaluation of available competing explanatory hypotheses can be made to collapse into an absolute evaluation of those hypotheses. In his second argument, Lipton argues that the Bad Lot Objection is incoherent because the assumption that we can find the best explanatory hypotheses in a given set entails that we are generally disposed to have made the true theory available. Finally, Schupbach's argument is that the Bad Lot Objection is simply misguided in that it faults IBE for failing to guarantee that 'good material content' is brought to the inferential table, whereas the mark of a cogent inference form is that it reliably preserves good material content; Schupbach's idea is that it is not the fault of IBE per se - understood as an inference form - that it should be possible to include only false hypotheses in the set of available, competing explanatory hypotheses from which an IBE is made.

In the bulk of this paper - sections 2, 3, and 4 - I discuss these reactionary responses to the Bad Lot Objection one by one, arguing that none of the responses undermines the objection. Although my conclusions in this paper are almost entirely negative, I will end each section with a somewhat more positive diagnosis of why these reactionary responses were destined to fail. Furthermore, my arguments strongly suggests that proponents of IBE would do well to focus their efforts on revisionary responses (rather than reactionary responses), i.e. on finding a more sophisticated characterization of IBE for which the Bad Lot Objection loses its bite. In the conclusion (section 5), I suggest that proponents of IBE have a great deal of work to do in this regard, and briefly discuss how a 'Peircean' conception of IBE avoids the Bad Lot Objection in an elegant way.

Before we begin, it is worth pointing out that although the Bad Lot Objection is often presented as a special problem for scientific realists - who often rely on IBE as the cornerstone of their epistemology (see, e.g., Psillos 1999) - it's clear that many anti-realists

3 Schupbach's response has already been endorsed by Peter Brössel (2015).

$4 \quad$ I am only aware of two other reactionary responses to the Bad Lot Objection - due to Psillos (1996) and Iranzo (2001) respectively. Both responses are closely related to Lipton's second response (discussed in section 3 below). 
should be equally concerned with van Fraassen's objection (including, arguably, van Fraassen himself). ${ }^{5}$ After all, the objection, if sound, would undermine the epistemic merits of IBE quite generally, regardless of whether it is being applied to observable everyday objects, or the theoretical posits of contemporary science. Indeed, the Bad Lot Objection is relevant to philosophical argumentation itself, which is increasingly (though often implicitly) based on employing IBE, complete with arguments that one's favorite philosophical theory should be accepted because it provides a better explanation than its currently available competitors. ${ }^{6}$ If the Bad Lot Objection cannot be dismissed, this piece of philosophical methodology may have to be reconsidered.

\section{Does Comparative Evaluation Collapse Into Absolute Evaluation?}

The first of Lipton's two arguments against the Bad Lot Objection aims to show that the comparative evaluations in which a hypothesis is taken to provide a better explanation than some other hypotheses collapses into an absolute (i.e. non-comparative) evaluation of each of the hypotheses involved in the comparison. Lipton starts by pointing out that we can always choose a set of available explanatory hypotheses that includes exactly two hypotheses, viz. the hypothesis we are concerned with, call it $\mathrm{H}_{\mathrm{i}}$, and its negation, not- $\mathrm{H}_{\mathrm{i}}$. When we compare $\mathrm{H}_{\mathrm{i}}$ and not- $\mathrm{H}_{\mathrm{i}}$, e.g. by determining that $\mathrm{H}_{\mathrm{i}}$ provides a better explanation than not$\mathrm{H}_{\mathrm{i}}$, we are in effect absolutely evaluating $\mathrm{H}_{\text {. }}$. So if IBE enables us to comparatively evaluate $H_{i}$ and not- $H_{i}$, then it also enables us to absolutely evaluate $H_{i}$. Note that this would not require us to consider the various more specific explanatory hypotheses with which $\mathrm{H}_{\mathrm{i}}$ competes, which is the difficulty that the Bad Lot Objection exploits. Lipton's initial idea, then, is that the possibility of considering hypotheses and their negations means that IBE can sidestep the Bad Lot Objection altogether.

Lipton recognizes that his opponent has a natural reply to this maneuver, viz. to argue that comparisons of mere contradictories (e.g. $\mathrm{H}_{\mathrm{i}}$ and not- $\mathrm{H}_{\mathrm{i}}$ ) are generally not

5 For a debate about whether van Fraassen's (1980) Constructive Empiricism is implicitly committed to a version of IBE (and is thus susceptible to the Bad Lot Objection in some form), see Psillos (1996), Ladyman et al. (1997), and Psillos (1997).

$6 \quad$ Not that there is any lack of explicit appeals to IBE in philosophical reasoning. For some recent examples, see Neta (2004), Williamson (2007), Sider (2008), Beebe (2009), and Paul (2012). 
feasible - that the comparisons with which IBE operates must generally be comparisons of more specific hypotheses that, while contraries to one another, are not mere contradictories. Let me elaborate on this briefly. For concreteness, consider the following run-of-the-mill example due to Jonathan Weisberg:

Suppose you come home one day to find the front door open and the lock broken. Furniture is overturned, the contents of the shelves are on the floor, and valuables are missing. One explanation is that someone broke in and stole your belongings, making a mess in the hurried process. (Weisberg 2009: 129-130)

Call the explanation proposed in the last sentence the break-in hypothesis. Now consider the negation of the burglar hypothesis - the no-break-in hypothesis - which simply claims that it is not the case that someone broke in, stole your belongings, and made a mess. Now, notice that this latter hypothesis provides no potential explanation at all of the various facts explained by the break-in hypothesis, e.g why the lock is broken and furniture is overturned. Indeed, given that the no-break-in hypothesis says only that one specific explanation for the state if your home is false, it is hard to see how it could possibly explain any of the facts explained by the break-in hypothesis at all. ${ }^{7}$ Thus, in so far as it makes sense to compare the explanatory qualities of the break-in hypothesis and its negation, IBE would trivially favor the former over the latter in virtue of the latter's inability to to provide any explanation of the relevant facts. (If this doesn't already strike you as absurd, notice that, by the same token, any hypothesis that provides a potential explanation of these facts would be trivially favored by IBE when compared with its negation.) In sum, then, the idea of producing absolute evaluations by forcing comparative evaluations of contradictories is itself susceptible to a problem that is at least as serious (and arguably more so) than the original Bad Lot Objection, viz. that IBE would generally seem to favor any explanatory hypothesis

7 It is important to see that it is not open to Lipton here to claim that not- $\mathrm{H}_{A}$ could be compared with $\mathrm{H}_{\mathrm{A}}$ in terms of how well various specific alternatives to $\mathrm{H}_{\mathrm{A}}$ that jointly constitute not- $\mathrm{H}_{\mathrm{A}}$ (in the sense of being conjunction in a long conjunction that is equivalent to not- $\mathrm{H}_{\mathrm{s}}$ ). After all, that would already require these various more specific alternatives to have been generated, which is what the Bad Lot Objection holds that we generally cannot do. 
over its negation due to the fact that the latter will often not provide any explanation of the relevant facts at all. ${ }^{8}$

Anticipating this response, Lipton goes on to argue that comparative evaluations collapse into absolute evaluations in a way that does not require a direct comparison between contradictories. The argument is nothing if not ingenious:

Suppose ... that we wish to rank the contradictories T1 and not-T1. If we find a contrary to T1 (say T2) that is ranked ahead of T1, then not-T1 is ranked ahead of T1, since T2 entails not-T1. Alternatively, if we find a contrary to not-T1 (say T3) that is ranked ahead of notT1, then T1 is ranked ahead of not-T1, since T3 entails T1. (Lipton 2004: 156)

This argument implicitly appeals to a principle that is worth making explicit for the subsequent discussion:

Lipton's Principle: If X should be ranked ahead of $\mathrm{Y}$, and $\mathrm{X}$ entails not-Y (because $\mathrm{X}$ and $\mathrm{Y}$ are contraries), then not-Y should be ranked ahead of $\mathrm{Y} .{ }^{9}$

Although Lipton's principle may be controversial, e.g. because a comparison of compatible hypotheses in the context of IBE seems to betray the idea that IBE chooses between competing explanatory hypotheses (Dellsén 2016), I will not criticize Lipton's Principle here. Nor will I explore whether Lipton's argument in the quoted passage is undermined by the fact that it presupposes that contraries to T1 and not-T1 (i.e. Lipton's T2 and T3 respectively) can be found - even though the whole point of the Bad Lot Objection is that the relevant contraries are often hard to find. My concern, rather, is whether Lipton's Principle would, if true, collapse comparative evaluations into absolute evaluations without

8 It is worth noting that the proponent of the Bad Lot Objection can concede that there may be are some cases in both a hypothesis and its negation provide (potential) explanations of the relevant facts, so that IBE does indeed provide comparisons of contradictories in those cases. After all, the Bad Lot Objection need not be seen as applicable absolutely all instances of IBE; it would suffice if it went through for a relatively large and/or salient class of IBEs - viz. those for which the negation of an explanatory hypothesis is too uninformative to provide a potential explanation of the relevant facts. Although I cannot myself think of a plausible example of this sort, I do invite others to try.

$9 \quad$ I take it that the motivation for Lipton's Principle is something along the following lines: If X entails not-Y, then $\mathrm{X}$ cannot be more probable than not-Y (this is a theorem of the probability calculus), so not-Y should be ranked above or level with $\mathrm{X}$. Given that IBE-ranking is transitive, it follows that if $\mathrm{X}$ should be ranked above $\mathrm{Y}$, not-Y should be ranked above $\mathrm{Y}$ as well. 
requiring the very same kind of comparisons that have already been shown to be highly problematic..$^{10}$

Notice first that in order for Lipton's argument to succeed, it would have to be possible to create (or recreate) the two kinds of scenarios Lipton imagines (involving T1, T2, and T3) for any theory that is being evaluated by IBE. After all, Lipton is arguing here that comparative evaluations collapse into absolute evaluations, so it wouldn't suffice to merely show that there are some cases in which an absolute evaluation can be achieved by means of a comparative evaluation. Accordingly, we must interpret Lipton here as presenting a general method for achieving absolute evaluations through comparative evaluations. A second thing worth noting is that Lipton actually presents two methods (or, if you prefer, two parts of one method) for absolutely evaluating T1 through comparative evaluations of contraries. The two methods are supposed to be complementary in that the first method delivers a verdict when not-T1 should be ranked above T1 (but is inconclusive when $\mathrm{T} 1$ should be ranked above not-T1), while the second method delivers a verdict when

10 As far as I am aware, the current literature on the Bad Lot Objection contains only two direct responses to this argument, given by Wray (2008) and Khalifa (2010) respectively. However, by my lights, Wray and Khalifa misinterpret Lipton in different ways, leading them to criticize less plausible versions of Lipton's argument. Although I cannot hope to do full justice to Wray's and Khalifa's responses here, let me nevertheless indicate here where my interpretation of Lipton's argument differs from theirs.

On Wray's reading of Lipton's argument, Lipton is appealing to the fact that for any two contraries $\mathrm{T} 1$ and T2, there will be some hypothesis $\mathrm{H} 1$ such that $\mathrm{T} 1$ entails $\mathrm{H} 1$ while T2 entails not-H1. Wray then goes on to argue that this does not allow us to infer that either T1 (or T2) "in all its details is likely true" (Wray 2008: 323, emphasis in original), only at most that an entailed consequence H1 (or not-H1) is true. However, this response seems to me to misrepresent Lipton's original argument. As Lipton presents his strategy, the aim is not merely to find some hypothesis H1 entailed by T1 and contradicted by T2. Rather, the point of the strategy is to get a ranking of a pair of contradictories $\mathrm{T} 1$ and not-T1, where there are no restrictions placed on what sort of theory T1 can be. Thus, as far as I can tell, there is no reason to think that Lipton's strategy would only allow us to infer a weaker hypothesis $\mathrm{H} 1$ that follows from T1.

Khalifa argues that Lipton's strategy would make scientists incoherentrankers. To show this, Khalifa starts by assuming that we are ranking T1 and not-T1, and that we've found (as per Lipton's strategy) a contrary to T1, viz. T2, that is ranked ahead of T1 so that (according to Lipton's principle) not-T1 would be ranked ahead of T1. Next Khalifa supposes that there is another contrary of T1 that is ranked below T1 (Khalifa calls this theory T3, though note that T3 would be a different theory than Lipton's T3, which is a contrary of not-T1). Khalifa then claims that "[s]ince T3 entails not-T1, once again invoking the quotation above [the same Lipton quotation I have provided on the previous pagel, T1 is ranked ahead of not-T1" (Khalifa 2010: 93). If Khalifa is right about this, then Lipton's strategy delivers the incoherent result that T1 should simultaneously be ranked both above and below not-T1. However, I cannot see any reason to attribute to Lipton the view that since T3 entails not-T1 (where T3 is a contrary to T1), T1 should be ranked above notT1. It certainly does not follow from Lipton's Principle, which is silent on whether the higher ranked of two contraries should be ranked above its contradictory. 
T1 should be ranked above not-T1 (but is inconclusive when not-T1 should be ranked above T1).

A third thing to notice is that it is only the second method that is of any real interest in the context of defending IBE against the Bad Lot Objection. To see why, notice that what we are interested in when we're worried about the Bad Lot Objection is the (absolute) epistemic status of the highest ranked available explanatory hypothesis; we are not, by contrast, interested in the status of any of the lower ranked hypotheses in a set of available competing explanatory hypotheses, since these have already been ruled out as noninferrable by IBE on account of their ranking below some other highest-ranked hypothesis. However, Lipton's first method can deliver absolute verdicts only about lower ranked contrary hypotheses, since it is part of the method itself to find a contrary explanatory hypothesis, viz. T2, that ranks above the hypothesis in question, viz. T1. To be sure, this would give us an absolute evaluation of $\mathrm{T} 1$ in virtue of indirectly enabling us to compare $\mathrm{T} 1$ and not-T1, but the fact that we would at that point have discovered T2, which ranks above T1, means that T1 would already be ruled out as uninferrable by IBE in virtue of ranking below T2. At that point, it would only be the absolute evaluation of T2 that would possibly be of interest in so far as we are concerned with the Bad Lot Objection, but notice that Lipton's method would have given us no way to make the relevant comparative evaluation of T2 and not-T2. Hence we have the somewhat paradoxical situation that this method applies only in cases in which the verdict it delivers is useless for the purposes of defending IBE against the Bad Lot Objection."

11 If you found that argument hard to follow, consider this example: A detective is investigating a murder, and has used IBE to determine that suspect number $i$ is the most plausible contender for having committed the crime (call this hypothesis $S_{\mathrm{i}}$ ). Having recently read about the Bad Lot Objection, our detective is worried that $S_{i}$ is merely the best of a bad lot of hypotheses, so she decides to use Lipton's first method to get an absolute (and not merely comparative) evaluation of this hypothesis. If she is successful at all, this will involve finding another competing hypothesis $S_{j}$ which is ranked above $S_{i}$ by IBE. At that point, however, her worry that $\mathrm{S}_{\mathrm{i}}$ is merely the best of a bad lot will have disappeared since $\mathrm{S}_{\mathrm{i}}$ is no longer the best of the competing explanatory hypotheses that are available to her. Rather, since $S_{\mathrm{i}}$ is (by assumption) better than $S_{\mathrm{i}}$, it is now $S_{\mathrm{i}}$ that she will worry is merely be the best of a bad lot. Since using Lipton's first method will have given her no way to absolutely evaluate $S_{\mathrm{i}}$, our detective would in effect be back where she started, again having to worry about whether the best available hypothesis (which has now gone from being $S_{\mathrm{i}}$ to being $\mathrm{S}_{\mathrm{i}}$ ) is merely the best of a bad lot. 
This leaves us with Lipton's second method. The idea behind this method is to rank T1 above not-T1 in virtue of ranking a contrary to not-T1, viz. T3, above not-T1. Lipton (implicitly) appeals to the fact that since T3 entails the negation of not-T1 (i.e. notnot-T1) it also entails T1 itself. Lipton's Principle then implies that if T3 should be ranked above not-T1, then T1 (which is entailed by T3) should be ranked above not-T1 as well. Hence Lipton's Principle would get us a comparison, i.e. a ranking, of the contradictories $\mathrm{T} 1$ and not-T1 via a comparison of the contraries T3 and not-T1. If this method really worked, it would indeed provide an absolute evaluation of a given theory via a comparison of contraries even in the more interesting case where we haven't already found a competing theory that is ranked above the theory in question.

However, this method is inapplicable for a different reason, viz. that we have been given no reason to think that a comparison between T3 and not-T1 is any more feasible than a comparison between $\mathrm{T} 1$ and not-T1. To see why, note that since T3 necessarily entails T1 (though presumably not vice versa, since that would make T1 and T3 equivalent) T3 is equivalent to a conjunction in which T1 is one of the conjuncts. In other words:

$$
\mathrm{T} 3 \equiv \mathrm{T} 1 \& \mathrm{P}
$$

where $\mathrm{P}$ is some proposition not entailed by $\mathrm{T} 1$. But if a comparison between a theory and its negation is not feasible in the first place, then clearly it is not feasible to compare the very same negation with a conjunction of the theory and some additional claim. Returning to our example of the the apparent burglary, Lipton's second method would have us rank the break-in hypothesis and its negation, the no-break-in hypothesis, via a comparison of the latter with a stronger version of the former, e.g. the break-in hypothesis conjoined with a claim about the exact time of the burglary (call this the timed-break-in hypothesis). However, if we couldn't already use IBE to compare the break-in hypothesis with its negation, the no-break-in hypothesis, then surely we will be no better off comparing the timed-break-in hypothesis with the no-break-in hypothesis. If anything, it is even more puzzling how these two hypotheses could be compared by IBE, since the former now makes a claim about the time of the supposed break-in that the latter hypothesis fails to even address. In sum, then, Lipton's second method does not provide a way to feasibly 
rank contradictories in the way that would be required for comparative evaluations to collapse into absolute evaluations as Lipton envisages.

Let me conclude this section by attempting to diagnose why Lipton's attempt to sidestep the Bad Lot Objection by collapsing comparative and absolute evaluations was destined to fail. The problem with Lipton's idea is not that ranking contraries is always feasible while ranking of contradictories never is, for we have seen that the problem is no less serious in a case of contraries that aren't contradictories, viz. in the comparison of the the no-break-in hypothesis with a strengthened version of the break-in hypothesis, the timed-break-in hypothesis. Rather, the problem is that hypotheses that are mere negations of genuinely explanatory hypotheses (e.g. the no-break-in hypothesis) generally just aren't the sort of hypotheses that could be feasibly compared in IBE with genuinely explanatory hypotheses (e.g. the break-in hypothesis). Hence, there is no general algorithm with which to generate a genuinely explanatory contradictory that could be compared in IBE so as to obtain an absolute evaluation of a given hypothesis. This is not to say that it is impossible to find cases in which a genuinely explanatory hypothesis and its negation can be feasible be compared (see footnote 9), but it does mean that Lipton's attempt to collapse comparative evaluations into absolute evaluations does not succeed.

\section{Does Reliable Ranking Entail Reliable Theory-Generation?}

Lipton's second argument against the Bad Lot Objection aims to show that there is a hidden incompatibility in the presuppositions of the objection. As Lipton points out, the Bad Lot Objection is meant to grant that scientists and others who use $\mathrm{IBE}^{12}$ are, at least generally, capable of reliably comparing hypotheses via IBE so that a more probable hypothesis is ranked above a less probable competitor. However, says Lipton, reliable ranking of this kind would be impossible unless relevant background theories were at least largely and/or approximately true, since false background theories "would skew the ranking, leading in

12 Judging by Lipton's formulations of his second response, it appears that he is exclusively or at least primarily concerned with whether scientists are susceptible to the Bad Lot Objection. Although Lipton's response does not seem to me to be restricted to any particular group of epistemic agents, I will nevertheless follow Lipton in formulating his response in terms of whether scientists are prone to generate approximately true theories. 
some cases to placing an improbable theory ahead of a probable competitor, and perhaps leading generally to true theories, when generated, being ranked below falsehoods." (Lipton 2004: 157) Lipton goes on to argue that these background theories could only be largely and/or approximately true if (approximately) true theories had a strong tendency to be generated in IBE, since the theories in question "are themselves the result of prior generation and ranking" (Lipton 2004: 157). Hence the assumption that scientists are reliable rankers of theories entails, according to Lipton, that they will tend to generate the relevant (approximately) true explanatory hypotheses, contrary to what the Bad Lot Objection presupposes. We can sum up this argument as follows:

P1. Scientists are reliable rankers of theories.

P2. If scientists are reliable rankers of theories, then their background theories are at least largely and/or approximately true.

P3. If scientists' background theories are largely and/or approximately true, then they generally generate approximately true theories.

C. So, scientists generally generate approximately true theories. [From P1, P2, and P3.]

If this argument is sound, it shows that proponents of the Bad Lot Objection cannot simultaneously concede that scientists are reliable rankers of theories and maintain that we generally have no reason to think that (approximately) true theories are among those that have been made available in IBE's first step.

Before we get to my main criticism of this argument, it is worth noting that P3 presupposes something that proponents of the Bad Lot Objection could reasonably reject, viz. that the relevant background theories are established by the same method of generation and comparative evaluation (namely, IBE) as the 'foreground' theories that scientists are assumed to be capable of reliably ranking in premise P1. But why should a proponent of the Bad Lot Objection, whose entire point is that IBE (as it is standardly formulated) is not a reliable form of ampliative inference, accept that our current background theories were established in that way? It is surely more natural for the proponent of the Bad Lot Objection to argue (as van Fraassen appears to do himself, e.g. in his 1989: 131-2) that IBE 
as standardly formulated is something philosophers have dreamed up in recent decades rather than the faithful description of scientists' actual inferential practices that proponents of IBE usually take it to be (e.g., McMullin 1992). So, in sum, premise P3 appears to beg an important question against at least some proponents of the Bad Lot Objection, viz. whether our background theories are in fact established by IBE.

However, my main criticism of Lipton's argument concerns not P3, but the combination of P1 and P2. Specifically, I submit that there is no reading of what it is for scientists to be 'reliable rankers' on which P1 and P2 are both plausibly true. To see this, consider two possible conceptions of what it is for someone to be a reliable ranker: ${ }^{13}$

(a) $\mathrm{S}$ is a reliable ranker iff, for a given pair of theories $\mathrm{H}_{\mathrm{i}}$ and $\mathrm{H}_{\mathrm{i}}, \mathrm{S}$ generally ranks $H_{i}$ above $H_{j}$ just in case $S$ 's total evidence provides more support for $H_{i}$ than for $H_{j}$.

(b) $\mathrm{S}$ is a reliable ranker iff, for a given pair of theories $\mathrm{H}_{\mathrm{i}}$ and $\mathrm{H}_{\mathrm{i}}, \mathrm{S}$ generally ranks $H_{i}$ above $H_{j}$ just in case $H_{i}$ is closer to the truth (i.e. more accurate) than $H_{j}{ }^{14}$

The difference between these two conceptions is that the ranking of theories is determined by scientists' evidential support on conception (a) whereas it is determined by closeness to the truth on conception (b). Since one's total evidence can be misleading, in the sense of providing more support for a less accurate theory, these two conceptions of rankingreliability are clearly not coextensive. The question, then, is whether Lipton's argument goes through on either of these conceptions. (I will argue that it doesn't.)

Conception (a) appears to be what proponents of the Bad Lot Objection have in mind when they are prepared to grant that scientists are reliable rankers of theories. Indeed, van Fraassen himself says that "the comparative judgment that this hypothesis is better than its actual rivals ... is indeed a 'weighing (in the light of) the evidence"' (van Fraassen 1989: 143). So premise P1 might indeed be acceptable given this conception of ranking-reliability, even to proponents of the Bad Lot Objection. However, P2 is quite clearly false on this

13 Lipton himself does not specify what is involved in being a reliable ranker of theories. Indeed, as far as I can see, Lipton uses these two conceptions interchangeably (Lipton 2004: 152, 157-159).

14. Note that one way in which $H_{i}$ can be closer to the truth than $H_{j}$ occurs in the special case when $H_{i}$ is precisely true and $\mathrm{H}_{\mathrm{j}}$ is not. 
conception. After all, the fact that scientists are generally able to correctly evaluate the relative support for two (or more) theories bestowed on them by their total evidence is perfectly compatible with all of their background theories being radically false (i.e. not even approximately true). This is just a corollary of the more general point that one's total evidence can be misleading - a point that is in turn analogous to the well-known fact that epistemically justified beliefs can be false. ${ }^{15}$ Of course, if one's background theories are indeed radically false, then the ability to reliably rank theories in this sense will not ensure that more accurate theories are ranked above their less accurate competitors. But that's perfectly compatible with P1 on conception (a), which says nothing about the relative closeness to truth of theories that are reliably ranked on this conception.

Consider next conception (b), which does view ranking-reliability as a matter of ranking theories in accordance with their relative closeness to the truth (i.e. their accuracy). On this conception, P2 is plausible since it seems that background theories must indeed generally be approximately true if the rankings of scientists are to be reliable in this sense. However, the issue here is that $\mathrm{P} 1$ is surely unacceptable on this conception. To see why, we need only consider cases in which scientists' total evidence is either misleading or lacking with regard to a comparison between two competing explanatory hypotheses $\mathrm{H}_{\mathrm{i}}$ and $\mathrm{H}_{\mathrm{j}}$. Consider, for example, a comparison in the early $19^{\text {th }}$ century between Wegener's theory of continental drift, and the received view of the continents as being fixed roughly where they are now - the fixed-continents theory. At this moment in time, the total evidence available to geologists arguably favored the fixed-continents theory, among other things because the available evidence indicated that the continents could not move as rapidly as Wegener's theory predicted in some important cases. It was not until the 1950s or 1960s that the weight of the evidence tipped in favor of Wegener's theory (or a version thereof,

15 Note that this is not just the familiar point (made in this context by Wray 2008, Khalifa 2010) that anti-realists such as van Fraassen will be skeptical about whether the explanatory considerations with which IBE operates, e.g. simplicity and explanatory scope, are genuinely epistemic reasons to believe that a hypothesis is true or whether they instead provide pragmatic reasons for believing or accepting these hypotheses. Rather, the point here is that an epistemic evaluation of a pair of hypotheses may be appropriate given the evidence even though that evaluation turns out to be incorrect in the sense that the favored hypothesis is false (or indeed further from the truth than the theory to which it is favorably compared). This point holds even if the one grants that explanatory considerations are genuinely epistemic reasons for belief. 
viz. plate tectonics), among other things because of evidence gathered with the help of new oceanographic technology (see, e.g., Bowler and Morus 2005: 237-252). However, since the theory of continental drift is clearly closer to the truth than the fixed-continents theory (or so we assume, at least for the sake of the example), early $19^{\text {th }}$ century geologists would have to rank the theory of continental drift above the fixed-continents theory for them to count as reliable rankers on conception (b) even though their evidence at the time clearly favored the latter over the former. More generally, conception (b) implies that reliable rankers have some sort of direct apprehension of the available theories' closeness to the truth, unmediated by their evidence at a given time. Since scientists clearly do not have abilities of this kind, premise P1 must be rejected on conception (b). It is even clearer that P1 so understood will not be found acceptable by anyone who is even remotely sympathetic to the Bad Lot Objection, and thus Lipton's attempt to show that the presuppositions of the objection are incoherent definitely fails on this conception.

To summarize: There is a conception of what it is to be a reliable ranker of theories that makes P1 true, viz. conception (a) on which reliable ranking is determined by the relative evidential support of the theories that are being ranked. However, on this conception, P2 is false since misleading evidence could easily lead to radically false background theories being ranked very highly. So Lipton's argument does not go through on conception (a). There is also a conception of ranking-reliability that makes $\mathrm{P} 2$ true, viz. conception (b) on which reliable ranking is determined by the relative closeness to the truth of the theories that are being ranked. However, on this conception, P1 is false since scientists do not have direct access to the relative accuracy of the theories that are being ranked. So Lipton's argument does not go through on conception (b) either. I thus conclude that there is no plausible way to uphold both of the relevant premises, P1 and P2, without equivocation in the definition of ranking-reliability. Furthermore, I conjecture that the prima facie plausibility of Lipton's argument is due to our own (understandable, though regrettable) tendency to equivocate in precisely this way, failing to distinguish between evaluations that match evidential support on the one hand, and closeness to the truth on the other. 


\section{Does IBE Nevertheless Preserve Good Material Content?}

Let us turn now to a more recent response to the Bad Lot Objection, one that is due to Jonah Schupbach (2014) and advocated in a similar context by Peter Brössel (2015). According to Schupbach, the Bad Lot Objection faults IBE for failing to guarantee that "good material content" is brought to the inferential table, whereas the mark of a cogent inference form is that it reliably "preserves good material content". (Schupbach 2014: 59) To see what Schupbach means by this, consider the basic form of IBE (as standardly formulated): ${ }^{16}$

P1. E.

P2. Among the available, competing explanatory hypotheses $\left\{\mathrm{H}_{1}, \ldots, \mathrm{H}_{\mathrm{n}}\right\}, \mathrm{H}_{\mathrm{i}}$ proffers the best potential explanation of $\mathbf{E}$.

C. $\mathrm{H}_{\text {. }}$.

Schupbach's idea is that in cases where we have a bad lot of hypotheses - i.e. a set $\left\{\mathrm{H}_{1}, \ldots, \mathrm{H}_{\mathrm{n}}\right\}$ in which all the hypotheses are false - the second premise in this inference form does not contain 'good material content'. However, Schupbach argues, it is not the fault of IBE per se - understood as an inference form - that it should be possible to include only false hypotheses in the set $\left\{\mathrm{H}_{1}, \ldots, \mathrm{H}_{\mathrm{n}}\right\}$ of available, competing explanatory hypotheses from which an IBE is made. That just shows that IBE, much like deductive inference forms such as modus ponens, cannot guarantee that it delivers good conclusions unless it is being fed good material from the outset.

Schupbach's argument relies on the distinction between an inference form and the material content that is being fed into that inference form in a particular instance. A sound criticism of IBE would have to show that IBE is faulty qua inference form, but Schupbach claims that the Bad Lot Objection shows no such thing. Instead, it shows only that it is possible for the conclusions one reaches via IBE to be unwarranted if one starts out by

16 This formulation of IBE is lifted verbatim from Schupbach (2014: 58) to ensure that we are operating with the exact same conception of IBE, except that I have used capitalized symbols for hypotheses and evidence. 
feeding IBE bad material content. But this cannot be a special problem for IBE since it is a feature of any inference for whatsoever:

[...] by virtue of their formal character, [inference forms] provide us with few constraints on the quality of the material that may be used to instantiate them on any occasion. [...] when working with bad material content, virtually any inference form will likely commend a false conclusion. (Schupbach 2014: 58)

Schupbach goes on to argue that we should evaluate IBE in the same way he claims we should evaluate any other inference form, i.e. by considering whether it "preserves good material content”:

When evaluating any inference form, we do not ask whether that form somehow guarantees the quality of the material content that we plug into it on any particular occasion. In and of itself, a decidedly cogent inference form may indeed give us no reason at all to expect that we will instantiate it with good material. Rather, we ask whether or not that inference form in some sense preserves good material content. (Schupbach 2014: 59 - emphasis in original)

However, Schupbach claims, the Bad Lot Objection does not even address the issue of whether IBE “preserves good material content” - it shows only that IBE, like virtually any inference form, delivers bad material content if it is fed bad material content. The upshot, then, is that the Bad Lot Objection is "just misguided" (Schupbach 2014: 55).

As this characterization of Schupbach's argument hopefully makes clear, a central notion in his defense of IBE against the Bad Lot Objection is the notion of preserving good material content. This is Schupbach's criterion of inferential cogency - what he claims that IBE is capable of doing reliably, the Bad Lot Objection notwithstanding. It is thus surprising and frustrating that Schupbach does not say explicitly what he means by this key piece of terminology. Some of what Schupbach says indicates that he is referring to a standard criterion for cogency of ampliative inferences - viz. that the conclusion should be likely to be true, justified, or known given that the premises are true, justified, or known. However, other remarks suggest that Schupbach is using a non-standard criterion of cogency for ampliative inferences which goes beyond a relationship between the premises and the conclusion of a given inference. Specifically, Schupbach suggests that inferences should be 
evaluated under the assumption that at least some of the hypotheses that are mentioned in their premises are true. In what follows, I discuss each possibility in turn, and argue that Schupbach's response fails to undermine the Bad Lot Objection on both standard and nonstandard conceptions of what it is to preserve good material content. Thus my argument in what follows can be presented as a dilemma: If Schupbach is using a standard criterion of cogency for ampliative inferences then his criticism of the Bad Lot Objection misses its mark, since (I shall argue) the Bad Lot Objection does show that IBE is not cogent according to standard criteria of inferential cogency. If, however, Schupbach is employing a non-standard criterion of cogency for ampliative inferences, then Schupbach's criticism is irrelevant to what the Bad Lot Objection was meant to show.

So suppose first that Schupbach means to be employing a standard criterion of cogency for ampliative inferences in his defense of IBE. Now, there is a well-known standard way of evaluating ampliative inference forms which is closely analogous to how we standardly evaluate deductive inference forms such as modus ponens. The idea is that just as we want deductive inferences to be such that it is impossible for the conclusion to be false if the premises are true, we want ampliative inferences to be such that it is improbable that the conclusion is false if the premises are true. Indeed, this standard way of evaluating ampliative inferences is adopted by Brian Skyrms (1986: 7) in an influential textbook on ampliative reasoning. According to Skyrms, an inference is inductively strong just in case the conclusion is (at least somewhat) likely to be true if the premises are true. ${ }^{17}$ Now, if IBE were inductively strong in Skyrms's sense, then there would certainly be a straightforward sense in which IBE “preserves good material content” as Schupbach claims it does. Indeed,

17 Although this criterion is crude and perhaps incomplete, it will do for our current purposes. Indeed, the discussion that follows assumes only that what Skyrms calls inductive strength is a necessary condition for ampliative cogency. This is important because there are reasons to think that inductive strength is not plausible as a sufficient condition for ampliative cogency, e.g. because an inference can be inductively strong in Skyrms's sense even if the premise(s) do not increase the probability of the conclusion at all. Consider, for example, an inference from 'it is raining' to 'the world is not going to end tomorrow'. Here, the conclusion is presumably very probably, but not thanks to the premises.

Skyrm's defintion of inductive strength also raises the issue of what kind of probability is involved. Presumably, the probability in question would have to be some kind of evidential or epistemic probability and this is indeed what Skyrms (1986: 15-20) takes it to be. I won't explore this issue further here since it is orthogonal to my main concerns. 
Schupbach's frequent comparisons between IBE and deductive inferences might suggest that this is what Schupbach is arguing. (Other remarks suggest a different interpretation, which we will consider below.)

However, IBE is not inductively strong, and a version of the Bad Lot Objection shows us why. Now, just as we can show that a form of inference is not deductively valid by showing that a single inference that takes this form has true premises and a false conclusion, we can show that a form of inference is not inductively strong by showing that a single inference that takes this form has true premises and a conclusion that is not likely to be true. ${ }^{18}$ So suppose some evidential statement $\mathbf{E}$ is true, and that some hypothesis $\mathrm{H}_{\mathrm{i}}$ provides the best potential explanation of $\mathbf{E}$ among a set of available, competing explanatory hypotheses $\left\{\mathrm{H}_{1}, \ldots, \mathrm{H}_{\mathrm{n}}\right\}$. This ensures that the two premises of IBE are both true in this case. But now suppose we have a "bad lot" of hypotheses in the sense that we have good reasons to believe that all of the available, competing explanatory hypotheses $\left\{\mathrm{H}_{1}, \ldots, \mathrm{H}_{\mathrm{n}}\right\}$ are false given our evidence $\mathrm{E}$. Then, no matter how well $\mathrm{H}_{\mathrm{i}}$ explains the relevant evidence $\mathrm{E}, \mathrm{H}_{\mathrm{i}}$ will be improbable given E. So we have that the premises of this IBE are both true while the conclusion $\mathrm{H}_{\mathrm{i}}$ is not likely to be true. It follows that IBE is not an inductively strong form of inference.

Let us illustrate this with a version of an example used for a different purpose by van Fraassen (1980: 19-20):

P1. I hear scratching in the wall, sounds of little feet pattering, and my cheese disappears.

P2. Among the available, competing explanatory hypotheses $\left\{H_{1}\right.$ : Someone is conducting an elaborate prank; $\mathrm{H}_{2}$ : I am hallucinating and losing my memory at the same time; $\mathrm{H}_{3}$ : The cheese has come to life and is roaming my walls\}, $\mathrm{H}_{1}$ proffers the best potential explanation of the evidence.

18 Of course, this would not show that we should never use the inference form in question, but rather only that the inference form cannot be trusted in all instances to take us from true premises to a probable conclusion. This is clearly what the Bad Lot Objection was meant to show. It was not meant to show, by contrast, that we can never go from true premises to a probable conclusion in IBE. 
C. $\mathrm{H}_{1}$ : Someone is conducting an elaborate prank.

To evaluate the inductive strength of this argument, let P1 and P2 be true. (Note that P2 can be true even if none of $\left\{\mathrm{H}_{1}, \mathrm{H}_{2}, \mathrm{H}_{3}\right\}$ are true.) Admittedly, it would be strange not to have considered the hypothesis that there is a mouse in the apartment, but if one hasn't considered that possibility, then $\mathrm{P} 2$ is still true. And yet I take it that $\mathrm{C}$ is very improbable in this case. So the premises are all true and yet the conclusion is not likely to be true. Hence IBE is not inductively strong.

So IBE does not "preserve good material content" in the sense of making the conclusion probable given the truth of the premises. ${ }^{19}$ One might think instead that "preserving good material content" is for Schupbach a matter of whether we have a reasonably good reason to believe the conclusion given that we have good reason to believe the premises. The idea would be that the "good material content" that is being "preserved" is justification or rationality of belief. However, IBE does not preserve good material content in this sense either, as should already be apparent from the discussion so far. After all, I may have excellent justification for premises P1 and P2 in the example above, and yet be utterly unjustified in believing the conclusion $\mathrm{C}$ on this basis. Put differently, the justification for premises P1 and P2 is not "preserved" through the inference to the conclusion $\mathrm{C}$ to any significant extent. This is most obvious if we also imagine that you have some special reasons for believing that the set of available, competing explanatory hypotheses does not contain a correct explanation, e.g. because your track record so far for coming up with correct explanations is very poor, or because a very trustworthy person tells you that all the explanations you have considered so far are false. But even in the original case, I think it should be clear that on any plausible conception of epistemic justification, one could be justified in believing both of the premises P1 and P2 (by perception and introspection respectively) and be utterly unjustified in believing the conclusion $\mathrm{C}$ on that

19 As a corollary, this also shows that IBE does not "preserve good material content" in the sense of it being probable that the conclusion is probable given that the premises are probable. 
basis. ${ }^{20}$ (An analogous argument shows that IBE does not "preserve good material content" in the sense of going from known premises to conclusions that are likely to be known.)

The key point here is that the available, competing hypotheses mentioned in the second premise of an IBE are not themselves premises of the inference. (If they were, then the conclusion would already occur in the premises, making IBE into a trivial kind of deductive inference.) Nor are they required to be true in order for the second premise to be true, as we have seen. The "bad lots" of the Bad Lot Objection are bad lots of available explanatory hypotheses, not bad lots of premises. With this is mind, we can say that what the Bad Lot Objection shows is that IBE can go from a good lot of premises to a bad conclusion, because we may have a bad lot of explanatory hypotheses in the second (good) premise. Since Schupbach's response does not undermine this point, it remains true that the Bad Lot Objection shows that IBE is not a cogent form of ampliative inference on the standard way of evaluating ampliative inferences in terms of their inductive strength.

A related point: Schupbach suggests that if the Bad Lot Objection were a problem for IBE, then it would be a problem for anyinference form whatsoever, including deductive inference forms such as modus ponens (Schupbach 2014: 55, 57-59, 63). According to Schupbach, this is because no form of inference could guarantee the quality of the "material content" that is fed into it (Schupbach 2014: 58). We are now in a position to see what is wrong with this argument: What the Bad Lot Objection shows is not that IBE fails to guarantee the quality of the premises brought to the inferential table, but that IBE can take us from true premises to conclusions that are not even likely to be true. In this respect, IBE differs from obviously cogent forms of inference such as modus ponens, where one clearly cannot go from true premises to an unlikely conclusion. The key point here is again that

20 Different conceptions of justification will give different rationales for this verdict. For example, a reliabilist will want to say that forming the belief that $\mathrm{C}$ is true on the basis of $\mathrm{P} 1$ and $\mathrm{P} 2$ does not amount to following a reliable belief-forming process (because the inductive rule in question does not reliably take one from true premises to true conclusions). An evidentialist, by contrast, will insist that $\mathrm{C}$ is not supported by the agent's evidence, which after all supports a competing hypothesis (that there is a mouse in the apartment) much more strongly. Of course, it may be possible to come up with entirely subjective conceptions of justification according to which the mere fact that you have only considered a certain set of competing explanations makes you justified in believing one of them, but $\mathrm{I}$ take it that no proponent of IBE (Schupbach included) would want to be saddled with having adopt such an extremely subjective view of justification in order to defend IBE against the Bad Lot Objection. 
the premises of an IBE can be true even though all the explanatory hypotheses mentioned in the second premise are false, in which case the IBE in question will go from true premises to a conclusion that is unlikely to be true. No similar possibility can arise for obviously cogent inference forms such as modus ponens. Hence the Bad Lot Objection does not generalize in the way Schupbach claims it does.

We turn now to the second horn of our dilemma for Schupbach's argument. So far I have shown that IBE is not an inductively strong form of inference, and is thus not inferentially cogent in any standard sense of taking one from true, justified, or known premises to conclusion that are likely to be true, justified, or known. Now, one could respond to this by adopting some alternative, non-standard conception of inferential cogency and argue that IBE is cogent in that sense, the Bad Lot Objection notwithstanding. Some of what Schupbach says suggests that this is the route he is taking, e.g. when he claims to have argued that "the goodness of IBE's material content is additionally [i.e., apart from its premises being truel a matter of whether the lot of hypotheses mentioned in IBE's second premise is good - i.e., whether the lot contains a true hypothesis" (Schupbach 2014: 62). The idea seems to be that "preserving good material content" - Schupbach's criterion for inferential cogency - does not concern whether the conclusion is likely to be true given the truth of the premises, but whether the conclusion is likely to be true given the truth of the premises and given the truth of at least some of the hypotheses that are mentioned in the premises.

Schupbach would certainly be right that if we use this as our criterion of inferential cogency for ampliative inferences, then the Bad Lot Objection would not show that IBE is not cogent. However, as we shall see shortly, this particular criterion is inadequate, since it implies that clearly fallacious inferences are cogent. Before we get to that, I want to discuss a more fundamental problem with this response - one that applies to any attempt to avoid the Bad Lot Objection by employing a non-standard conception of inferential cogency. The problem, in short, is that it is not clear why anyone should feel satisfied with the result that IBE is cogent according to a non-standard criterion of inferential cogency. What we care about is whether IBE takes us from premises that are true, justified, or known to conclusions that are likely to be true, justified, or known. If we must admit that IBE does 
not do this (as I argued in the previous section), then why should we care whether there is some other non-standard criterion of inferential cogency on which IBE is cogent?

Indeed, it would certainly be unfair to the proponent of the Bad Lot Objection perhaps even question-begging - to complain that the objection fails to show that IBE is not cogent in some non-standard sense. It is worth noting that van Fraassen himself was almost certainly employing a standard criterion of cogency when he presented the Bad Lot Objection in Laws and Symmetry (1989). In the relevant passage, van Fraassen (1989: 142143) argues that even on the assumption that $H_{i}$ provides the best explanation of some evidence $\mathrm{E}$ among the available hypotheses $\left\{\mathrm{H}_{1}, \ldots, \mathrm{H}_{\mathrm{n}}\right\}$, the conclusion $\mathrm{H}_{\mathrm{i}}$ could still fail to be "more likely to be true, than not". (1989: 143) This is most naturally interpreted as criticizing IBE for failing to guarantee that its conclusion is likely to be true given that both of its premises are true. So even if Schupbach succeeds in showing that IBE is cogent in his preferred sense, this appears to be irrelevant to the objection van Fraassen presented, since there is no reason to think that his objection aimed to establish that IBE is not cogent in Schupbach's non-standard sense.

As mentioned above, there is a second problem with defending IBE against the Bad Lot Objection by using Schupbach's non-standard criterion of cogency for ampliative inferences. The problem, in short, is that the criterion that Schupbach seems to adopt viz. that an inductive inference is cogent iff the conclusion is likely to be true given the truth of the premises and given the truth of at least some of the hypotheses mentioned in the premises - counts clearly fallacious inference forms as cogent. To see why, consider the following inference form:

P1. So far, source $\mathrm{S}$ has proposed hypotheses $\left\{\mathrm{H}_{1}, \ldots, \mathrm{H}_{\mathrm{n}}\right\}$.

P2. $H_{1}, \ldots, H_{n-1}$ have all been refuted.

\section{C. $\mathrm{H}_{\mathrm{n}}$.}

If an inductive argument is cogent whenever the conclusion is probable given the truth of the premises and given the truth of at least one of the hypotheses mentioned in the premises, then this argument is cogent. To see why, suppose P1 and P2 are true and that at least one of the hypotheses $\left\{\mathrm{H}_{1}, \ldots, \mathrm{H}_{\mathrm{n}}\right\}$ mentioned in $\mathrm{P} 1$ is true. Since $\mathrm{H}_{\mathrm{n}}$ would be the only remaining 
hypothesis in $\left\{\mathrm{H}_{1}, \ldots, \mathrm{H}_{\mathrm{n}}\right\}$ not refuted according to $\mathrm{P} 2, \mathrm{H}_{\mathrm{n}}$ would at least be very likely to be true. ${ }^{21}$ However, this is clearly a very poor form of ampliative inference. Indeed, notice that this inference form essentially recommends trusting a source that has so far only proposed hypotheses that have been refuted.

It is also worth noting that the requirement that at least some of the hypotheses mentioned in an argument's premises be true clearly does not apply to deductive inferences. The standard criterion of cogency for deductive inferences is validity, i.e. that the conclusion is guaranteed to be true if the premises are true. By contrast, it is not a criterion of cogency for deductive inferences that the conclusion is guaranteed to be true if the premises are true and if at least some of the hypotheses mentioned in the premises are true. ${ }^{22}$ So the requirement that we evaluate inferential cogency under the assumption that at least some of the hypotheses mentioned in the premises are true (in addition to assuming that the premises are true) clearly cannot be generalized to deductive inferences. Contrary to what Schupbach suggests (2014: 57-59), the kind of cogency that applies to ampliative inferences such as IBE would have to be of a very different kind than the one that applies to deductive inferences (and not just because IBE, like any ampliative inference form, does not guarantee the truth of its conclusions given the truth of its premises).

To sum up, Schupbach's main contention is that the Bad Lot Objection faults IBE for not guaranteeing the quality of the content that is fed into it. On Schupbach's view, IBE does "preserve good material content" and is thus a cogent form of ampliative inference. I have argued that Schupbach's response faces a dilemma: On the first horn, we evaluate IBE by employing a standard criterion of cogency for ampliative inferences on which such

21 Depending on what we read into the statement that $H_{1}, \ldots, H_{n-1}$ have been 'refuted', $H_{n}$ may even be guaranteed to be true.

22 Indeed, this criterion would lead to absurd results. For example, the following fallacious piece of reasoning would be cogent qua deductive inference:

P. If $\mathrm{H}_{1}$ then $\mathrm{H}_{2}$.

C. $\mathrm{H}_{2}$.

To see why, suppose $\mathrm{P}$ is true and that at least one of $\mathrm{H}_{1}$ and $\mathrm{H}_{2}$ is true. Now, if $\mathrm{H}_{1}$ is true, then by $\mathrm{P}, \mathrm{H}_{2}$ is true as well. So $\mathrm{C}$ is true in that case. But if $\mathrm{H}_{2}$ is true then $\mathrm{C}$ is also true. Thus $\mathrm{C}$ is true in either case. So $\mathrm{C}$ is guaranteed to be true given the truth of $\mathrm{P}$ and the truth of at least one of the hypotheses that are mentioned in $\mathrm{P}$. 
an inference is cogent if its conclusion is likely to be true, justified, or known given that its premises that are true, justified, or known. I have shown that IBE is not cogent by this criterion and thus does not "preserve good material content" in any straightforward sense. On the second horn of the dilemma, we evaluate IBE by employing a non-standard criterion of cogency for ampliative inferences. However, the criterion of cogency for ampliative inferences to which Schupbach sometimes seems to be appealing cannot be correct, since it implies that clearly fallacious inferences would be cogent. Moreover, the criterion does not generalize to deductive inferences, contrary to what Schupbach suggests. Finally, and most importantly, even if Schupbach's criterion were adequate, it still would do nothing to undermine van Fraassen's original Bad Lot Objection, since there is no reason to think that van Fraassen's objection was employing Schupbach's non-standard criterion of cogency.

\section{Concluding Remarks}

In section $1, \mathrm{I}$ distinguished between reactionary and revisionary responses to the Bad Lot Objection. In the sections that followed, I argued that that none of the three most prominent reactionary responses to the objection is successful. This strongly suggests that revisionary responses are more promising, and thus that proponents of IBE should focus their attention on finding a more sophisticated characterization of IBE for which the Bad Lot Objection loses its bite. A popular way of achieving this has been to strengthen the conditions under which IBE licenses inference. In particular, many have followed Alan Musgrave (1988), who suggested that in order for a hypothesis to be inferable by IBE the explanation it provides must be satisfactory, i.e. sufficiently good, in addition to being the best among those provided by available hypotheses. ${ }^{23}$ Despite its popularity, however, Musgrave's suggestion

23 Somewhat paradoxically, Lipton $(2004: 56,63,154)$ sometimes seems to endorse this suggestion, although it is unclear to what extent this is meant as a response to the Bad Lot Objection. Indeed, in his discussion of the Bad Lot Objection, Lipton explicitly mentions the possibility of insisting "that scientists are capable of absolute and not only comparative evaluation" (which is what an evaluation of an explanation as "sufficiently good" would require). He goes on to say that "[t]hese responses may well be correct but, baldly asserted, they lead to an unsatisfying standoff between those who believe in absolute evaluation ... and those who do not" (Lipton 2004: 154), before presenting the two reactionary responses I discussed in sections 2 and 3. 
has never been fleshed out with an anything resembling an account of what it is for an explanation to be "satisfactory," so the current status of IBE is at best unclear on Musgrave's suggestion. ${ }^{24}$

My own view is that the Bad Lot Objection calls for a different kind of revisionary response - one on which IBE is modified by weakening its conclusion. Specifically, I argue elsewhere (Dellsén MS) that the primary function of IBE is to help identify which explanatory hypotheses of those available it would be advisable to adopt as the working hypotheses around which further investigation is structured. This represents a partial return to some of Charles S. Peirce's $(1934,1935)$ ideas about explanatory reasoning (what he usually called 'abduction'), in that Peirce describes 'abduction' as warranting only a probative kind of acceptance for the purposes of inductive examination (Kapitan 1992: 1217). Now, while the possibility of having a 'bad lot' of available hypotheses means that the risk of adopting a false proposition as a working hypotheses can often be significant, the Bad Lot Objection does not undermine IBE on this conception since an agent can do no better than to let the best of the available hypotheses guide her further investigations. Although I haven't defended this view of IBE in this paper, I do hope to have shown that one of the problems that the view is meant to address - viz. the Bad Lot Objection - is a genuine epistemic difficulty that be brushed aside as fallacious, incoherent, or misguided. ${ }^{25}$

24 The problem here is not just the familiar one of proving accounts of the explanatory considerations with which IBE operates - e.g. consilience, parsimony, elegance, fertility, testability - and how these considerations interact in an overall comparison between the explanatory goodness (i.e. loveliness) of different hypotheses. Musgrave's suggestion additionally requires that there be some absolute measure of each explanatory consideration, and some way of determining an absolute degree of explanatory goodness for every hypothesis in logical space.

25 I am grateful to Hrafn Asgeirsson and Elanor Taylor for insightful comments on earlier drafts of this paper. I would also like to thank three anonymous reviewers for this journal for criticisms and suggestions that helped to improve the paper in various respects. 


\section{References}

Beebe, J. R. (2009). "The Abductivist Reply to Skepticism”, Philosophy and Phenomenological Research 79: 604-636.

Bowler, P., and Morus, I. R. (2005). Making Modern Science: A Historical Survey. Chicago and London: University of Chicago Press.

Brössel, P. (2015). "On the Role of Explanatory and Systematic Power in Scientific Reasoning”, Synthese 192: 3877-3913.

Carrier, M. (1991). "What is wrong with the miracle argument?", Studies in History and Philosophy of Science 22: 23-36.

Chakravartty, A. (2008). "What You Don’t Know Can’t Hurt You: Realism and the Unconceived”, Philosophical Studies 137: 149-58.

Dellsén, F. (2016). "Explanatory Rivals and the Ultimate Argument”, Theoria 82: 217-237.

Dellsén, F. (forthcoming). "Realism and the Absence of Rivals", Synthese. DOI: 10.1007/s11229-(016-1059-3.

Dellsén, F. (MS). "The Heuristic Conception of Inference to the Best Explanation”, unpublished manuscript.

Devitt, M. (2011). “Are Unconceived Alternatives a Problem for Scientific Realism?”, Journal for General Philosophy of Science 42: 285-93.

Douven, I. (1999). "Inference to the Best Explanation Made Coherent", Philosophy of Science (Proceedings) 66: S424-S435.

Douven, I. (2013). "Inference to the Best Explanation, Dutch Books, and Inaccuracy Minimisation”, Philosophical Quarterly 63: 428-444.

Egg, M. (2016). "Expanding Our Grasp: Causal Knowledge and the Problem of Unconceived Alternatives”, British Journal for the Philosophy of Science 67: 115-141.

Harman, G. (1965). "The Inference to the Best Explanation”, The Philosophical Review, 74: 88-95. 
Henderson, L. (2014). "Bayesianism and Inference to the Best Explanation”, British Journal for the Philosophy of Science 65: 687-715.

Iranzo, V. (2001). “Bad Lots, Good Explanations”, Critica: Revista Hispanoamericana de Filosofia 33: 71-96.

Kapitan, T. (1992). "Peirce and the Autonomy of Abductive Reasoning”, Erkenntnis 37: $1-26$.

Khalifa, K. (2010). "Default Privilege and Bad Lots: Underconsideration and Explanatory Inference”, International Studies in the Philosophy of Science 24: 91-105.

Ladyman, J., Douven, I., Horsten, L., and van Fraassen, B. C. (1997). "A Defense of Van Fraassen's Critique of Abductive Inference: Reply to Psillos”, The Philosophical Quarterly 47: 305-321.

Laudan, L. (1981). “A Confutation of Convergent Realism”, Philosophy of Science 48: 1949 .

Lipton, P. (1993). "Is the Best Good Enough?”, Proceedings of the Aristotelian Society 93: 89-104.

Lipton, P. (2004). Inference to the Best Explanation (2 ed.). London and New York: Routledge.

Magnus, P. D. (2010). "Inductions, Red Herrings, and the Best Explanation for the Mixed Record of Science”, British Journal for the Philosophy of Science 61: 803-19.

McMullin, E. (1992). The Inference That Makes Science. Milwaukee, WI: Marquette University Press.

Musgrave, A. (1988). "The Ultimate Argument for Scientific Realism”. In R. Nola (ed.), Relativism and Realism in Science, pp. 229-252. Dordrecht and Boston: Kluwer Academic Publishers.

Neta, R. (2004). "Skepticism, Abductivism, and the Explanatory Gap", Philosophical Issues 14: $296-325$.

Okasha, S. (2000). "Van Fraassen’s Critique of Inference to the Best Explanation”, Studies in History and Philosophy of Science 31: 691-710. 
Paul, L. A. (2012). “Metaphysics as Modeling: The Handmaiden’s Tale”, Philosophical Studies 160: 1-29.

Peirce, C. S. (1934). The Collected Papers of Charles Sanders Peirce (Vol. 5). Cambridge, MA: Harvard University Press.

Peirce, C. S. (1935). The Collected Papers of Charles Sanders Peirce (Vol. 6). Cambridge, MA: Harvard University Press.

Poincaré, H. (1952). Science and Hypothesis. New York: Dover. Republication of the first English translation, published by Walter Scott Publishing, London, 1905.

Psillos, S. (1996). “On Van Fraassen's Critique of Abductive Reasoning”, The Philosophical Quarterly 46: 31-47.

Psillos, S. (1997). "How Not to Defend Constructive Empiricism: A Rejoinder", The Philosophical Quarterly 47: 369-72.

Psillos, S. (1999). Scientific Realism: How Science Tracks Truth. London: Routledge.

Schupbach, J. N. (2014). "Is the Bad Lot Objection Just Misguided?”, Erkenntnis 79: 5564.

Sider, T. (2008). "Introduction", in Sider, T., J. Hawthorne, and D. Zimmerman (eds.), Contemporary Debates in Metaphysics (Malden, MA: Blackwell), pp. 1-8.

Skyrms, B. (1986). Choice and Chance: An Introduction to Inductive Logic. Belmont, CA: Wadsworth.

Sklar, L. (1981). "Do Unborn Hypotheses Have Rights?” Pacific Philosophical Quarterly 62: 17-29.

Stanford, P. K. (2006). Exceeding Our Grasp: Science, History, and the Problem of Unconceived Alternatives. Oxford: Oxford University Press.

Thagard, P. (1989). "Explanatory Coherence”, Behavioral and Brain Sciences 14: 435-502. Thagard, P. (1991). "The Dinosaur Debate: Explanatory Coherence and the Problem of Competing Hypotheses”. In Cummins, R. and Pollock, J. (eds.), Philosophy and AI: Essays as the Interface, pp. 279-300. Cambridge, MA: MIT Press. 
van Fraassen, B. C. (1980). The Scientific Image. Oxford: Clarendon Press.

van Fraassen, B. C. (1989). Laws and Symmetry. Oxford: Clarendon Press.

Weisberg, J. (2009). "Locating IBE in a Bayesian Framework”, Synthese 167: 125-143.

Williamson, T. (2007). The Philosophy of Philosophy. Malden, MA: Blackwell.

Wray, K. B. (2008). "The Argument From Underconsideration as Grounds for Anti-

Realism: A Defense", International Studies in the Philosophy of Science 22: 317-326.

Wray, K. B. (2011). "Epistemic privilege and the success of science”, Nous 46: 375-385. 